\title{
CORRECTION
}

\section{Endovascular treatment of abdominal aortic aneurysms}

Dominique B. Buck, Joost A. van Herwaarden, Marc L. Schermerhorn and Frans L. Moll

Nat. Rev. Cardiol. 11, 112-123 (2014); doi:10.1038/nrcardio.2013.196

In the version of this article originally published, the following statement was missing from the

acknowledgements: This work was supported by the NIH T32 Harvard-Longwood Research Training in Vascular

Surgery grant HLO07734. This error has been corrected in the HTML and PDF versions of the article. 